\title{
Notas Científicas
}

\section{Controle químico de doenças foliares na cultura do arroz irrigado}

\author{
Alisson Celmer ${ }^{(1)}$, Marcelo Gripa Madalosso ${ }^{(1)}$, Mônica Paula Debortoli(1), \\ Lucas Navarini ${ }^{(1)}$ e Ricardo Silveiro Balardin ${ }^{(1)}$
}

\begin{abstract}
(1)Universidade Federal de Santa Maria, Dep. de Defesa Fitossanitária, Caixa Postal 5.025, CEP 97111-970 Santa Maria, RS.E-mail: alissoncelmer@yahoo.com.br,mgmadalosso@yahoo.com.br,mpdebortoli@yahoo.com.br, navarini@gmail.com, rsbalardin@balardin.com.br
\end{abstract}

\begin{abstract}
Resumo - O objetivo deste trabalho foi avaliar a interferência das doenças foliares na produtividade de duas cultivares de arroz irrigado, bem como determinar a interação entre ingredientes ativos e momento de aplicação, para o controle de escaldadura e mancha-marrom. Foram utilizados: tiofanato metílico, tebuconazole, azoxystrobin e trifloxystrobin + propiconazole, aplicados em cinco épocas, às cultivares IRGA 417 e El Passo L 144. Os parâmetros avaliados foram: severidade das doenças e rendimento de grãos. O controle químico proporcionou redução significativa na severidade das doenças, em ambas as cultivares, com reflexo no rendimento de grãos, e foi influenciado pelo produto e pela época de aplicação.
\end{abstract}

Termos para indexação: Oryza sativa, Bipolaris oryzae, Monographella albescens, fungicidas, época de aplicação, produtividade.

\section{Chemical control of irrigated rice diseases}

\begin{abstract}
The objective of this work was to evaluate the influence of leaf diseases on the yield of two rice varieties, and to determine the interaction between active ingredients and application stages, for the control of brown leaf spot and oat scald. The products used were: thiofanate methylic, tebuconazole, azoxystrobin and trifloxystrobin + propiconazole, applied in five stages, on the varieties IRGA 417 and El Passo L 144. The parameters observed were disease severity and yield. Chemical control reduced the diseases severity in both cultivars, wich reflected on yield, and was influenced by product e application stage.
\end{abstract}

Index terms: Oryza sativa, Bipolaris oryzae, Monographella albescens, fungicides, application stage, yield.

Na Região Sul do Brasil, a cultura do arroz irrigado é acometida por várias doenças, que podem prejudicar a produtividade e a qualidade dos grãos colhidos (Sociedade..., 2003). Segundo Balardin \& Borin (2001), os danos no rendimento causados por manchas foliares podem ser de até $50 \%$. Os danos mais significativos, no Sul do Brasil, têm sido relacionados à incidência de mancha-marrom ou mancha-parda (Bipolaris oryzae (Breda de Haan) Shoem) e escaldadura (Monographella albescens (Thümen) Parkinson, Sivanesan \& C. Booth). Também tem sido observada, mas de forma secundária ou localizada, a incidência de mancha-das-bainhas, mancha-estreita e mancha-de-alternária.

A escaldadura é uma doença comum nas regiões de cultivo de arroz (Ou, 1987), e tem sido observada freqüentemente nas regiões Centro-Oeste, Norte brasileiro e litoral paranaense, sob condições de alta temperatura e alta umidade relativa do ar (Prabhu \& Filippi, 1997; Balardin
\& Borin, 2001). No Rio Grande do Sul, a doença surgiu nas últimas décadas, com ataques leves, que têm se intensificado, comportamento que, segundo Marzari et al (2007), pode ocorrer em razão da troca das cultivares tradicionais pelas modernas e do lançamento de cultivares especialmente tolerantes a brusone, o que condicionou o surgimento das demais doenças foliares, como escaldadura, mancha-parda e mancha-das-glumas, que, até então, eram consideradas doenças secundárias na cultura do arroz.

Os sintomas das doenças são observados nas folhas, colmos e panículas, com maior intensidade nas fases de perfilhamento e emborrachamento (Filippi \& Prabhu, 1998).

A mancha-marrom, também citada na literatura como mancha-parda, é uma das principais doenças causadoras de manchas nos grãos, e pode causar danos de 12 a $30 \%$ no peso dos grãos, e de 18 a $22 \%$ no número de grãos cheios por panícula, além de causar chochamento e perda de qualidade, por causa do gessamento e coloração escura 
(Prabhu \& Filippi, 1997), e pode reduzir a emergência em razão da morte de plântulas (Ribeiro \& Sperandio, 1998). Os sintomas da mancha-marrom são manchas foliares ovais de cor marrom, que nos grãos aparecem como manchas marrom-escuras.

Diversos estudos têm sido conduzidos com o objetivo de verificar a resposta ao controle químico de doenças na cultura do arroz. A aplicação de fungicidas de parte aérea tem se mostrado uma medida eficaz para controle dessas doenças, como observado por Ottoni et al (2000), que obtiveram controle eficiente de mancha-parda pela aplicação de fungicidas na cultivar Maravilha. A aceitação dessa prática pelos produtores deve-se à maior estabilidade na produção, aumento da qualidade de grãos e ao consistente retorno econômico.

Este trabalho teve por objetivo avaliar a interferência das doenças foliares na produtividade de duas cultivares de arroz irrigado, bem como determinar a interação entre ingredientes ativos e momento de aplicação, para o controle de escaldadura e mancha-marrom.

O experimento foi conduzido no Município de Camaquã, RS, com as cultivares IRGA 417 e El Passo L 144, semeadas no sistema de semeadura direta. Os tratos culturais seguiram as recomendações técnicas da cultura do arroz irrigado (Sociedade..., 2003). A área do experimento foi dessecada com herbicida glifosato na dose de $2 \mathrm{~L} \mathrm{ha}^{-1}$. A densidade populacional foi aproximadamente de 3 milhões de plantas por ha, com espaçamento entre linhas de $0,17 \mathrm{~m}$, e adubação de base de $200 \mathrm{~kg} \mathrm{ha}^{-1}$ de NPK (5-20-30). A adubação de cobertura foi fracionada em três aplicações de $50 \mathrm{~kg} \mathrm{ha}^{-1}$ de uréia, nos estádios fenológicos de início do perfilhamento, emborrachamento e no início da emissão de panículas. A irrigação ocorreu aos 20 dias após a emergência, e foi mantida lâmina permanente de água de $0,15 \mathrm{~m}$ de altura, durante o período de execução do experimento.

Os tratamentos testados foram quatro fungicidas: tiofanato metílico (350 $\mathrm{g} \mathrm{ha}^{-1}$ de i.a.); tebuconazole (120 $\mathrm{g} \mathrm{ha}^{-1}$ de i.a.); trifloxystrobin + propiconazole (150 $\mathrm{g} \mathrm{ha}^{-1}$ de i.a.); e azoxystrobin (100 $\mathrm{g} \mathrm{ha}^{-1}$ de i.a.). Os fungicidas foram aplicados em diferentes estádios de desenvolvimento da cultura: final do perfilhamento, emborrachamento, emissão de panículas, final do perfilhamento + emborrachamento e emborrachamento + emissão de panículas. O delineamento experimental foi o inteiramente casualizado, em arranjo fatorial $(5 \times 4 \times 2)$, cujos fatores foram cinco épocas de aplicação de fungicida, quatro ingredientes ativos e duas cultivares, com quatro repetições. A significância dos fatores foi determinada por meio da análise de variância. Foi utilizado o teste de Tukey para comparação das médias.

As aplicações dos fungicidas foram realizadas com pulverizador costal pressurizado a $\mathrm{CO}_{2}$, acoplado a uma barra com quatro pontas Teejet XR 11002, espaçadas em $0,5 \mathrm{~m}$, e com funcionamento à pressão de $30 \mathrm{lb} \mathrm{pol}^{-2}$, tendo-se aplicado volume de calda de $200 \mathrm{~L} \mathrm{ha}^{-1}$. No momento da pulverização, a temperatura e velocidade do vento eram inferiores a $28^{\circ} \mathrm{C}$ e $3 \mathrm{~km} \mathrm{~h}^{-1}$, respectivamente, e umidade relativa do ar superior a $50 \%$.

Cada unidade experimental apresentou área total de $12 \mathrm{~m}^{2}(2 \times 6 \mathrm{~m})$, tendo sido considerados $7,5 \mathrm{~m}^{2}$ como área útil, com descarte da bordadura da parcela $(0,25 \mathrm{~m}$ em cada lado e $0,5 \mathrm{~m}$ em cada cabeceira). $\mathrm{Na}$ área útil, foi realizada a avaliação da severidade das doenças e colheita para cálculo do rendimento de grãos.

Foram realizadas avaliações aos 15, 30 e 45 dias após a primeira aplicação, correspondentes aos estádios de emissão da panícula, florescimento e enchimento de grãos, respectivamente. A avaliação considerou a folha-bandeira, bandeira-1 e bandeira-2 de todas as plantas da área útil da unidade experimental, em duas tomadas de notas por parcela. A avaliação dos tratamentos, quanto à eficácia do controle, foi obtida pela consideração da severidade (porcentagem de tecido foliar com sintomas) de mancha-marrom (B. oryzae) e de escaldadura (M. oryzae).

O rendimento dos grãos foi obtido da área útil da parcela onde foram colhidas todas as plantas, trilhadas em batedor estacionário; a massa de grãos foi pesada e foi determinada a umidade, que após convertida a 13\%, possibilitou o cálculo da produção por hectare.

Houve interação significativa entre os fatores avaliados (cultivares, ingredientes ativos e épocas de aplicação), tanto em relação à severidade das doenças como ao rendimento de grãos; entretanto, foi observado um efeito maior de cultivares e dos ingredientes ativos sobre o rendimento de grãos, e da época de aplicação sobre a severidade das doenças.

A severidade de mancha-marrom, na cultivar IRGA 417, foi afetada pela época de aplicação. A aplicação realizada no final do perfilhamento resultou em maior severidade que as aplicações realizadas nos estádios mais tardios. Nesse caso, a época mais adequada para realizar o controle da doença foi na emissão da panícula com trifloxystrobin + propiconazole; resultado semelhante foi obtido no controle da escaldadura (Tabela 1). Segundo Prabhu \& Filippi (1997), as manchas foliares manifestam-se durante ou logo após a fase da floração. Deste modo, em aplicações precoces, os fungicidas podem não proporcionar efeito residual suficiente para proteger a planta até a maturação. 
Na cultivar El Passo L 144, para controle de manchamarrom, os fungicidas tebuconazole, aplicado no emborrachamento, e tiofanato metílico, aplicado no final do perfilhamento + emborrachamento e emborrachamento + emissão de panículas, proporcionaram melhor desempenho. Observando-se o controle da escaldadura, os fungicidas tebuconazole e trifloxystrobin + propiconazole foram mais eficientes, quando aplicados no emborrachamento, o que pode ser explicado pelo fato de esta doença ter seu ataque mais expressivo nesse estádio fenológico (Filippi et al., 2004). Os resultados corroboram os de Filippi et al. (2005), que obtiveram redução significativa na incidência de escaldadura, pela aplicação de tebuconazole em arroz de terras altas.

O rendimento de grãos de ambas as cultivares foi influenciado pelo controle químico das doenças foliares. $\mathrm{Na}$ cultivar El Passo L 144, foi observado incremento de produtividade de $3.434 \mathrm{~kg} \mathrm{ha}^{-1}$ pela aplicação de trifloxystrobin + propiconazole, no final do perfilhamento + emborrachamento; na cultivar IRGA 417, o acréscimo de produtividade foi de $2.538 \mathrm{~kg} \mathrm{ha}^{-1}$, quando aplicado trifloxystrobin + propiconazole, no emborrachamento + emissão de panículas (Tabela 2). Esses resultados demonstram que o controle de doenças pode ser considerado uma importante ferramenta, para manutenção da estabilidade de produção de grãos da cultura do arroz irrigado. Segundo Marzari et al. (2007), a diminuição da severidade de doenças, pelo controle químico, propicia o aumento da produtividade, fatos semelhantes aos encontrados por Slaton et al. (2003) e Fageria \& Prabhu (2004), que verificaram aumento na produtividade com a aplicação de fungicidas para controle de doenças em arroz.

Os resultados demonstraram o potencial de dano das doenças foliares, que podem impactar negativamente a produção de grãos, pois foram observadas reduções superiores a $3 \mathrm{t} \mathrm{ha}^{-1}$, na produtividade do tratamento testemunha, com relação aos tratamentos, nas cultivares avaliadas (Tabela 2).

Com base nos resultados, pode-se inferir que a escolha do ingrediente ativo, somada à época de aplicação adequada, tem efeito direto no controle de mancha-marrom e escaldadura, e na produtividade das cultivares de arroz irrigado El Passo L 144 e IRGA 417.

O controle químico pode ser considerado estratégia essencial de manejo na cultura do arroz irrigado, para a maximização do ganho no rendimento.

Tabela 1. Severidade de escaldadura (Monographella albescens) e mancha-marrom (Bipolaris oryzae), nas cultivares El Passo L 144 e IRGA 417, submetidas à aplicação de fungicidas, nos estádios de desenvolvimento do final do perfilhamento (FP), emborrachamento (E), emissão de panículas (EPa), final do perfilhamento + emborrachamento (FP+E), e emborrachamento + emissão de panículas $(\mathrm{E}+\mathrm{EPa})^{(1)}$.

\begin{tabular}{|c|c|c|c|c|}
\hline \multirow[t]{2}{*}{ Tratamento } & \multicolumn{2}{|c|}{ El Passo L 144} & \multicolumn{2}{|c|}{$\begin{array}{c}\text { IRGA } 417 \\
\end{array}$} \\
\hline & Escaldadura & Mancha-marrom & Escaldadura & Mancha-marrom \\
\hline Tiofanato metílico/FP & $2,56 \mathrm{bc}$ & 1,33 bcde & $5,05 \mathrm{gh}$ & $2,70 \mathrm{i}$ \\
\hline Tiofanato metílico/E & $2,26 \mathrm{ab}$ & $1,17 \mathrm{abcd}$ & $9,94 \mathrm{i}$ & $2,16 \mathrm{gh}$ \\
\hline Tiofanato metílico/EPa & $2,93 \mathrm{bcd}$ & $1,23 \mathrm{abcd}$ & 4,70defgh & $1,81 \mathrm{ef}$ \\
\hline Tiofanato metílico/FP+E & $2,60 \mathrm{bc}$ & $1,07 \mathrm{ab}$ & $5,19 \mathrm{~h}$ & $2,00 \mathrm{fg}$ \\
\hline Tiofanato metílico/E+EPa & $2,67 \mathrm{bcd}$ & $1,09 \mathrm{ab}$ & $4,77 \mathrm{efgh}$ & $1,91 \mathrm{fg}$ \\
\hline Tebuconazole/FP & $2,78 \mathrm{bcd}$ & $1,11 \mathrm{abc}$ & $5,25 \mathrm{~h}$ & $2,37 \mathrm{hi}$ \\
\hline Tebuconazole/E & $2,12 \mathrm{a}$ & $1,01 \mathrm{a}$ & $3,77 \mathrm{bcde}$ & $1,52 \mathrm{~cd}$ \\
\hline Tebuconazole/EPa & $2,69 \mathrm{bcd}$ & 1,16abcd & $3,06 \mathrm{ab}$ & $1,16 \mathrm{ab}$ \\
\hline Tebuconazole/FP+E & $2,37 \mathrm{abc}$ & $1,35 \mathrm{bcde}$ & $5,27 \mathrm{hi}$ & $2,02 \mathrm{fg}$ \\
\hline Tebuconazole/E+EPa & $2,78 \mathrm{bcd}$ & $1,34 \mathrm{abcde}$ & $4,08 \mathrm{cdefgh}$ & $1,42 \mathrm{~cd}$ \\
\hline Trifloxystrobin + propiconazole/FP & $2,85 \mathrm{bcd}$ & $1,52 \mathrm{def}$ & $3,83 \mathrm{bcdef}$ & $1,86 f$ \\
\hline Trifloxystrobin + propiconazole/E & $2,82 \mathrm{bcd}$ & $1,38 \mathrm{bcde}$ & $3,00 \mathrm{ab}$ & $1,39 \mathrm{~cd}$ \\
\hline Trifloxystrobin + propiconazole/EPa & $2,85 \mathrm{bcd}$ & $1,27 \mathrm{abcde}$ & $2,65 \mathrm{a}$ & $1,02 \mathrm{a}$ \\
\hline Trifloxystrobin + propiconazole/FP+E & $2,53 \mathrm{bc}$ & $1,55 \mathrm{ef}$ & $3,62 \mathrm{bcd}$ & $1,56 \mathrm{~d}$ \\
\hline Trifloxystrobin + propiconazole/E+EPa & $2,78 \mathrm{bcd}$ & $1,61 \mathrm{ef}$ & $3,63 \mathrm{bcd}$ & $1,37 \mathrm{~cd}$ \\
\hline Azoxystrobin/FP & $3,02 \mathrm{bcd}$ & $1,43 \mathrm{cdef}$ & 4,31defgh & $2,18 \mathrm{gh}$ \\
\hline Azoxystrobin/E & $2,82 \mathrm{bcd}$ & 1,34 bcde & 4,12 defgh & $1,58 \mathrm{de}$ \\
\hline Azoxystrobin/EPa & $3,20 \mathrm{~cd}$ & $1,80 \mathrm{~g}$ & $3,90 \mathrm{bcdefg}$ & $1,31 \mathrm{bc}$ \\
\hline Azoxystrobin/FP+E & $2,96 \mathrm{bcd}$ & $1,69 \mathrm{fg}$ & $3,62 \mathrm{bcd}$ & $1,44 \mathrm{~cd}$ \\
\hline Azoxystrobin/E+EPa & $3,40 \mathrm{~d}$ & $1,68 \mathrm{fg}$ & $3,13 \mathrm{abc}$ & $1,42 \mathrm{~cd}$ \\
\hline Testemunha & $12,00 \mathrm{e}$ & $12,84 \mathrm{~h}$ & $10,80 \mathrm{i}$ & $14,81 \mathrm{j}$ \\
\hline $\mathrm{CV}(\%)$ & 9,12 & 10,39 & 5,61 & 4,81 \\
\hline
\end{tabular}

${ }^{(1)}$ Médias seguidas por letras iguais, na coluna, não diferem entre si pelo teste de Tukey, a $5 \%$ de probabilidade. 
Tabela 2. Rendimento de grãos das cultivares de arroz irrigado El Passo L 144 e IRGA 417, submetidas à aplicação de fungicidas, nos estádios de desenvolvimento do final do perfilhamento (FP), emborrachamento (E), emissão de panículas (EPa), final do perfilhamento + emborrachamento (FP+E), e emborrachamento + emissão de panículas $(\mathrm{E}+\mathrm{EPa})^{(1)}$.

\begin{tabular}{|c|c|c|}
\hline \multirow[t]{2}{*}{ Tratamento } & \multicolumn{2}{|c|}{ Rendimento de grãos $\left(\mathrm{kg} \mathrm{ha}^{-1}\right)$} \\
\hline & El Passo L 144 & IRGA 417 \\
\hline Tiofanato metílico/FP & $5.456 \mathrm{ab}$ & $5.561 \mathrm{ab}$ \\
\hline Tiofanato metílico/E & $6.602 \mathrm{abcd}$ & $6.3304 \mathrm{ab}$ \\
\hline Tiofanato metílico/EPa & $6.433 \mathrm{abcd}$ & $5.957 \mathrm{ab}$ \\
\hline Tiofanato metílico/FP+E & 6.396abcd & $6.340 \mathrm{ab}$ \\
\hline Tiofanato metílico/E+EPa & $6.423 \mathrm{abcd}$ & $6.486 \mathrm{ab}$ \\
\hline Tebuconazole/FP & $5.706 \mathrm{abc}$ & $6.727 \mathrm{ab}$ \\
\hline Tebuconazole/E & $5.605 \mathrm{abc}$ & $6.496 \mathrm{ab}$ \\
\hline Tebuconazole/EPa & $5.613 \mathrm{abc}$ & $6.816 \mathrm{ab}$ \\
\hline Tebuconazole/FP+E & $7.388 \mathrm{bcd}$ & $6.408 \mathrm{ab}$ \\
\hline Tebuconazole/E+EPa & $7.518 \mathrm{bcd}$ & $6.164 \mathrm{ab}$ \\
\hline Trifloxystrobin + propiconazole/FP & $7.582 \mathrm{bcd}$ & $6.222 \mathrm{ab}$ \\
\hline Trifloxystrobin + propiconazole/E & $7.718 \mathrm{~cd}$ & $6.210 \mathrm{ab}$ \\
\hline Trifloxystrobin + propiconazole/EPa & $7.972 \mathrm{~d}$ & $6.496 \mathrm{ab}$ \\
\hline Trifloxystrobin + propiconazole $/ \mathrm{FP}+\mathrm{E}$ & $8.344 \mathrm{~d}$ & $6.700 \mathrm{ab}$ \\
\hline Trifloxystrobin + propiconazole $/ \mathrm{E}+\mathrm{EPa}$ & $7.591 \mathrm{~cd}$ & $7.336 b$ \\
\hline Azoxystrobin/FP & $6.656 \mathrm{abcd}$ & $6.600 \mathrm{ab}$ \\
\hline Azoxystrobin/E & $7.456 \mathrm{bcd}$ & $6.085 \mathrm{ab}$ \\
\hline Azoxystrobin/EPa & $8.092 \mathrm{~d}$ & $6.064 \mathrm{ab}$ \\
\hline Azoxystrobin/FP+E & $7.171 \mathrm{bcd}$ & $6.691 \mathrm{ab}$ \\
\hline Azoxystrobin/E+EPa & $7.054 \mathrm{bcd}$ & $6.907 \mathrm{ab}$ \\
\hline Testemunha & $4.909 \mathrm{a}$ & $4.798 \mathrm{a}$ \\
\hline $\mathrm{CV}(\%)$ & 11,53 & 12,70 \\
\hline
\end{tabular}

${ }^{(1)}$ Médias seguidas por letras iguais, em cada cultivar, não diferem entre si pelo teste de Tukey, a 5\% de probabilidade.

\section{Referências}

BALARDIN, R.S.; BORIN, R.C. Doenças na cultura do arroz irrigado. Santa Maria: [s.n], 2001. 48p.il.

FAGERIA, N.K.; PRABHU, A.S. Controle de brusone e manejo de nitrogênio em cultivo de arroz irrigado. Pesquisa Agropecuária Brasileira, v.39, p.123-129, 2004.

FILIPPI, M.C.; PRABHU, A.S. Doenças do arroz e seu controle. In: BRESEGHELLO, F.; STONE, L.F. Tecnologias para o arroz de terras altas. Santo Antônio de Goiás: Embrapa Arroz e Feijão, 1998. p.139-156.

FILIPPI, M.C.; PRABHU, A.S.; SILVA, G.B. da. Cultivo de arroz irrigado no Estado de Tocantins. Sistemas de Produção, n.3. Embrapa Feijão e Arroz, 2004. Disponível em: http:// sistemaproducao.cnptia.embrapa.br/FontesHTML/Arroz/ ArrozIrrigadoTocantins/.

FILIPPI, M.C.; PRABHU, A.S.; SILVA, G.B. da. Escaldadura do arroz e seu controle. Santo Antônio de Goiás: Embrapa Arroz e Feijão, 2005. 4p. (Circular Técnica, 72).

MARZARI, V.; MARCHEZAN, E.; SILVA, L.S. da; CAMARGO, E.R.; TELÓ, G.M. População de plantas, dose de nitrogênio e aplicação de fungicida na produção de arroz irrigado: I - características agronômicas. Ciência Rural, v.37, p.330-336, 2007.
OTTONI, G.; OLIVEIRA, W.F.; SILVA, A.L.; ALBERNAZ, K.C.; SILVA, E.G.; CARDOSO, E.; GUICHERIT, E. Eficiência de fungicidas no controle de mancha-parda (Bipolaris oryzae) em arroz (Oryza sativa). Pesquisa Agropecuária Tropical, v.30, p.59-62, 2000.

OU, S.H. Rice diseases. $2^{\text {nd }}$ ed. Slough: CAB International Mycological Institute, 1985. 380p.

PRABHU, A.S.; FILIPPI, M.C. Arroz (Oryza sativa L.) Controle de doenças. In: VALE, F.X.R.; ZAMBOLIN, L. Controle de doenças de plantas: grandes culturas. Viçosa: Universidade Federal de Viçosa, 1997. v.1. p.51-79.

RIBEIRO, A.S.; SPERANDIO, C.A. Controle de doenças na cultura do arroz irrigado. In: PESKE, S.T.; NEDEL, J.L.; BARROS, A.C.S.A. Produção de arroz irrigado. Pelotas: Universidade Federal de Pelotas, 1998. p.301-349.

SLATON, N.A.; CARTWRIGHT, R.D.; MENG, J.; GBUR, E.E.; NORMAN, R.J. Sheath blight severity and rice yield as affected by nitrogen fertilizer rate, application method and fungicide. Agronomy Journal, v.95, p.1489-1496, 2003.

SOCIEDADE SUL-BRASILEIRA DE ARROZ IRRIGADO (Sosbai). Arroz irrigado: recomendações técnicas da pesquisa para o Sul do Brasil. Itajaí: Sosbai, 2003. 126p. 